\title{
An Extremal Characterization of Projective Planes
}

\author{
Stefaan De Winter* \\ Department of Mathematics and Computer Algebra \\ Ghent University, 9000 Gent, Belgium \\ sgdwinte@cage.ugent.be \\ Felix Lazebnik ${ }^{\dagger}$ \\ Department of Mathematical Sciences \\ University of Delaware, Newark, DE 19716, USA \\ lazebnik@math.udel.edu \\ Jacques Verstraëte ${ }^{\ddagger}$ \\ Department of Mathematics \\ University of California, San Diego, 9500 Gilman Drive, La Jolla California 92093-0112, USA \\ jacques@ucsd.edu
}

Submitted: Jul 22, 2008; Accepted: Nov 20, 2008; Published: Nov 30, 2008

Mathematics Subject Classification: 05B25, 05C35, 05C38, 51E14, 90C30

\begin{abstract}
In this article, we prove that amongst all $n$ by $n$ bipartite graphs of girth at least six, where $n=q^{2}+q+1 \geq 157$, the incidence graph of a projective plane of order $q$, when it exists, has the maximum number of cycles of length eight. This characterizes projective planes as the partial planes with the maximum number of quadrilaterals.
\end{abstract}

\section{Introduction}

The problem of maximizing the number of copies of a graph $H$ in an $F$-free graph has been investigated at length by numerous researchers (see, for example, Fisher [9] and Györi, Pach and Simonovits [13], Fiorini and Lazebnik [7, 8]). The Turán problem is the most familiar instance of this problem, where $H=K_{2}$, and is discussed in detail in Bollobás [2], Füredi [10], Simonovits [18]. To mention another example, Erdős [5] conjectured that the

\footnotetext{
${ }^{*}$ The author is a Postdoctoral Fellow of the Research Foundation Flanders.

${ }^{\dagger}$ This research was partially supported by the NSA, Grant H98230-08-1-0041.

${ }^{\ddagger}$ This research was supported by an Alfred P. Sloan Research Fellowship and the NSF, Grant DMS 0800704 .
} 
maximum number of cycles of length five in an $n$-vertex triangle-free graph is achieved by the blowup of a pentagon (see Györi [12] for details).

In order to present our results, we will need the following definitions and notations. Any graph-theoretic notion not defined here may be found in Bollobás [3]. All our graphs are finite, simple and undirected. If $G=(V, E)=(V(G), E(G))$ is a graph, then the order of $G$ is $|V|$, the number of vertices of $G$, and the size of $G$ is $|E|$, the number of edges in $G$. For a vertex $v \in V, N(v)=N_{G}(v)=\{u \in G: u v \in E\}$ denotes the neighborhood of $v$, and $d(v)=d_{G}(v)=|N(v)|$ - the degree of $v$. The minimum degree and maximum degree of $G$ are denoted $\delta(G)$ and $\Delta(G)$. If the degrees of all vertices of $G$ are equal $d$, $G$ is called $d$-regular. For a graph $F$, we say that $G$ is $F$-free if $G$ contains no subgraph isomorphic to $F$. A $k$-cycle is a cycle of length $k$, i.e., a cycle with $k$ edges. We denote by $c_{k}(G)$ the number of $k$-cycles of $G$. The girth of a graph $G$ containing cycles, denoted by $g=g(G)$, is the length of a shortest cycle in $G$. By $G(A, B ; E)$ we denote a bipartite graph with $A$ and $B$ representing the parts of $G$. When $|A|=m$ and $|B|=n$, we refer to $G$ as an $m$ by $n$ bipartite graph. A partial plane $\pi=(\mathcal{P}, \mathcal{L} ; I)$ is an incidence structure with a set of points $\mathcal{P}$, a set of lines $\mathcal{L}$, and a symmetric binary relation of incidence $I \subseteq(\mathcal{P} \times \mathcal{L}) \bigcup(\mathcal{L} \times \mathcal{P})$ such that any two distinct points are on at most one line, and every line contains at least one point. The definition implies that any two lines share at most one point. (Our definition of a partial plane is more general than the usual one, where every line is required to contain at least two points.) The Levi graph of a partial plane $\pi$ is its point-line bipartite incidence graph $G(\pi)=G(\mathcal{P}, \mathcal{L} ; E)$, where $x y \in E$ if and only if point $x$ is on line $y$. The Levi graph of any partial plane is 4-cycle-free.

A generalized $k$-gon of order $(q, q)$, for $k \geq 3$ and $q \geq 2$, denoted $\Pi_{q}^{k}$, is a partial plane whose Levi graph is a $(q+1)$-regular graph of girth $2 k$ and diameter $k$. It is easy to argue that in such a graph each partition contains $n_{q}^{k}=q^{k-1}+q^{k-2}+\ldots+q+1$ vertices (for information on generalized polygons, see Van Maldeghem [19] or Brouwer, Cohen and Neumaier [4]). In the case $k=3$, when the geometry is better known as a projective plane of order $q$, we write $\Pi_{q}=\Pi_{q}^{3}$ and $n_{q}=n_{q}^{3}$. It follows from a theorem by Feit and Higman [6] that if $\Pi_{q}^{k}$ exists, then $k \in\{3,4,6\}$. For each of these $k, \Pi_{q}^{k}$ are known to exist only for arbitrary prime power $q$.

In this paper, we are interested in studying the maximum possible number of $2 k$-cycles in an $n$ by $n$ bipartite graph of girth $g$. When $g=4$, the maximum is, clearly, $\frac{(k !)^{2}}{2 k}\left(\begin{array}{l}n \\ k\end{array}\right)^{2}$, and is attained only by $K_{n, n}$ - the complete $n$ by $n$ bipartite graph.

It was shown in [8] that the maximum number of 6-cycles in an $n_{q}$ by $n_{q}$ bipartite graph of girth six is achieved only by a $G\left(\Pi_{q}\right)$. This gives an extremal characterization of projective planes as the partial planes with a maximum number of triangles - pairs of three distinct points and three distinct lines, where the points represent pairwise intersections of the lines.

For 2-connected bipartite graphs of girth $2 k$, Teo and Koh [16] gave an upper bound on the number of $2 k$-cycles which is monotone increasing in the size of the graph, and 
which coincides with the number of cycles of length $2 k$ in a $G\left(\Pi_{q}^{k}\right)$. A consequence of the main result in Hoory [11] is that $G\left(\Pi_{q}^{k}\right)$ have the greatest size among all $n_{q}^{k}$ by $n_{q}^{k}$ bipartite graphs of girth $2 k$, when $k \in\{3,4,6\}$. For $k=3$, the result has appeared in Reiman [17] (or see Bollobás [2]), and for $k=4$, an independent proof appeared in Neuwirth [15]. This implies that the problem of maximizing the number of $2 k$-cycles is completely solved in these cases.

The next instance of the problem is to maximize the number of cycles of length $g+2$ in an $n$ by $n$ bipartite graph of girth $g$. It was shown in [7], that any $n_{q}$ by $n_{q}$ bipartite graph of girth at least six achieving the maximum number of 8-cycles has average degree in the interval $(q-1, q+1]$. It was also conjectured there that if $\Pi_{q}$ exists, then the average degree of such a graph is $q+1$. In this case it is of the greatest size among all 4-cycle-free $n_{q}$ by $n_{q}$ bipartite graphs. Therefore it must be isomorphic to a $G\left(\Pi_{q}\right)$. We confirm this conjecture by proving the following theorem.

Theorem 1 Let $n=n_{q}=q^{2}+q+1 \geq 157$, and suppose that $\Pi_{q}$ exists. Then, for any $n$ by $n$ bipartite graph $G$ of girth at least six,

$$
c_{8}(G) \leq c_{8}\left(G\left(\Pi_{q}\right)\right)
$$

with equality if and only if $G=G\left(\Pi_{q}\right)$.

This theorem characterizes projective planes as the partial planes with a maximum number of quadrilaterals (a precise definition of a quadrilateral will be given later). To make the upper bound in Theorem 1 explicit, we determine $c_{8}\left(G\left(\Pi_{q}\right)\right)$. Thinking in terms of $\Pi_{q}$, one can construct all cycles of length eight in $G\left(\Pi_{q}\right)$ by first choosing two lines, which will contain a pair of opposite sides, and then choosing a pair of points on each of them distinct from the point of intersection of these lines. Four chosen points are vertices of two distinct quadrilaterals with a pair of opposite sides on the chosen lines. Clearly every quadrilateral in the projective plane (equivalently, every 8-cycle in $G\left(\Pi_{q}\right)$ ) is constructed via this procedure exactly twice. Therefore

$$
c_{8}\left(G\left(\Pi_{q}\right)\right)=\left(\begin{array}{c}
n_{q} \\
2
\end{array}\right)\left(\begin{array}{l}
q \\
2
\end{array}\right)^{2} .
$$

\section{Proof of Theorem 1}

We begin by tightening the aforementioned result from [7] concerning the average degree. This will allow us to obtain the lower bound of 157 on $n$ in Theorem 1. Using the original result from [7] would yield the lower bound of 254 .

Lemma 2.1 Let $q \geq 3$ be a positive integer, $n=n_{q}=q^{2}+q+1$, and suppose that $\Pi_{q}$ exists. Let $G=G(A, B ; E)$ be an $n$ by $n$ bipartite graph of girth at least six having the maximum number of 8-cycles. Then the average degree of $G$ is in $(q-0.05, q+1]$. 
Proof. All ideas and results we need to prove the statement are already in [7]. According to [7, (2.4) on page 195], the number $p_{3}(G)$ of paths of length three in $G$ is at most $n g\left(\frac{e}{n}\right)$, where $g(x)=x(n-x)$, i.e.,

$$
p_{3}(G) \leq e(n-e / n) .
$$

The inequalities [7, (2.6)-(2.7b) on page 196] give

$$
8 c_{8}(G) \leq(e-2 n+1) p_{3}(G) .
$$

As $G$ has at least as many 8-cycles as the Levi graph of a projective plane of order $q$, $c_{8}(G) \geq\left(\begin{array}{l}n \\ 2\end{array}\right)\left(\begin{array}{c}q \\ 2\end{array}\right)^{2}$, and so

$$
\frac{1}{8}(e-2 n+1) e\left(n-\frac{e}{n}\right) \geq\left(\begin{array}{l}
n \\
2
\end{array}\right)\left(\begin{array}{l}
q \\
2
\end{array}\right)^{2} .
$$

It is very easy to verify that this implies the lemma. The details are left to the reader. The constant 0.05 is not optimal, and can be decreased, e.g., to 0.03. As such an improvement requires some additional explanation and will not effect the subsequent results (nor the lower bound on $n$ in Theorem 1), we do not pursue it.

The following lemma bounds the maximum degree in an $n$ by $n$ bipartite graph of girth at least six having the greatest number of 8-cycles. The result will be essential for obtaining an upper bound on the number of 8-cycles in such a graph in Lemma 2.3.

Lemma 2.2 Let $q \geq 12$ be a positive integer, $n=n_{q}=q^{2}+q+1$, and suppose that $\Pi_{q}$ exists. Let $G=G(A, B ; E)$ be an $n$ by $n$ bipartite graph of girth at least six having the greatest number of 8-cycles, and let $\Delta$ be its maximum degree. Then $\Delta<\frac{n}{4}$.

Proof. By a result in [17], the number of edges in an $m$ by $n$ bipartite graph without 4 -cycles, $m \leq n$, is at most

$$
\frac{n}{2}+\sqrt{\frac{n^{2}}{4}+n m^{2}-n m} .
$$

Let $d_{G}(x)=\Delta$, and $\Delta \geq \frac{n}{4}$. We may assume $x \in A$. Let $A^{\prime}=A \backslash\{x\}$, and $B^{\prime}=B \backslash N_{G}(x)$. Deleting $x$ and $N_{G}(x)$ from $G$, we obtain a bipartite graph $G^{\prime}=G^{\prime}\left(A^{\prime}, B^{\prime} ; E^{\prime}\right)$ with $\left|A^{\prime}\right|=n-1$ and $\left|B^{\prime}\right|=n-\Delta \leq 3 n / 4$. Let $e=e(G)$ and $e^{\prime}=e\left(G^{\prime}\right)$. Since $G$ contains no 4-cycles, any vertex from $A^{\prime}$ is adjacent to at most one vertex from $N_{G}(x)$. Hence, $e \leq e^{\prime}+\Delta+(n-1) \leq e^{\prime}+2 n-1$. As $G^{\prime}$ contains no 4-cycles, the upper bound (1), being an increasing function of $m$ on $[1 / 2, n]$, gives

$$
e^{\prime} \leq \frac{n-1}{2}+\sqrt{\frac{(n-1)^{2}}{4}+(n-1) \frac{9 n^{2}}{16}-(n-1) \frac{3 n}{4}},
$$

and hence

$$
\frac{e}{n} \leq \frac{10 n-6+\sqrt{9 n^{3}-17 n^{2}+4 n+4}}{4 n} .
$$


By Lemma 2.1, $\frac{e}{n}>q-0.05$. Therefore

$$
\frac{10 n-6+\sqrt{9 n^{3}-17 n^{2}+4 n+4}}{4 n}>q-0.05 .
$$

It is easy to check, however, that this is false for all $n \geq 12^{2}+12+1=157$, and the lemma is proved.

In what follows we prefer to use the geometric terminology. Let $\mathcal{P}$ and $\mathcal{L},|\mathcal{P}|=|\mathcal{L}|=n$ denote the points and lines of the partial plane $\pi$. If a point $Y$ lies on line $x$ we will write $Y \in x$. If $X$ and $Y$ are collinear (distinct) points we write $X \sim Y$, and by $X Y$ we denote the line passing through them. The number of points on a line $x$ is denoted by $d(x)$. We define a 4-tuple in $\pi$ as a sequence of its four distinct points. A 4-gon in $\pi$ is a 4-tuple ( $A, B, C, D)$, with the property that $A \sim B \sim C \sim D \sim A$, and such that no three of these points are collinear. We assume that eight distinct 4-gons

$$
\begin{aligned}
& (A, B, C, D),(B, C, D, A),(C, D, A, B),(D, A, B, C), \\
& (D, C, B, A),(C, B, A, D),(B, A, D, C),(A, D, C, B)
\end{aligned}
$$

give rise to the same quadrilateral in $\pi$, i.e, the same 8-cycle in the corresponding Levi graph $G$ of $\pi$, which is completely defined by its set of vertices and its set of edges.

If $c_{4}(\pi)$ denotes the number of quadrilaterals in $\pi$, then the number of 4 -gons is $8 c_{4}(\pi)$.

For integers $n \geq r \geq 1$, let $n_{(r)}$ denote the product $n(n-1) \cdots(n-r+1)$. We claim that the following holds.

Lemma 2.3 Let $\pi=(\mathcal{P}, \mathcal{L}, I)$ be a partial plane with $|\mathcal{P}|=|\mathcal{L}|=n=n_{q}=q^{2}+q+1$, and with the greatest number of quadrilaterals. Let $G=G(\pi)$ be its Levi graph. Then

$$
c_{8}(G)=c_{4}(\pi) \leq \frac{1}{8} \sum_{x \in \mathcal{L}} d(x)_{(2)}(n-d(x))_{(2)}-\frac{1}{4} \sum_{x \in \mathcal{L}} d(x)_{(3)}(n-d(x)) .
$$

Proof. The first sum $\sum_{x \in \mathcal{L}} d(x)_{(2)}(n-d(x))_{(2)}$ in the right hand side of (2) counts the number of 4-tuples $(A, B, C, D)$ such that $A \sim B$, and neither $C$ nor $D$ is on the line $A B$. The second sum $\sum_{x \in \mathcal{L}} d(x)_{(3)}(n-d(x))$ counts the number of 4-tuples $(A, B, C, D)$ such that $A, B, C$ are on a line and $D$ is off this line. Hence, no 4 -tuple is counted by both sums. Clearly each 4-gon is counted by the first sum exactly once, and it is not counted by the second sum at all. It is also clear that the first sum counts also some 4-tuples which are not 4-gons. We will show that the number of those is at least twice as large as the value of the second sum, and this will prove (2).

In order to do this, we consider the following four classes $\mathcal{C}_{i}, i=0,1,2,3$, of configurations in $\pi$, where the class $\mathcal{C}_{i}$ is formed by the sets $\{A, B, C, D\}$ of four distinct points such that three of them are collinear, the fourth is off the line defined by these three and is collinear with exactly $i$ of them. 
We begin with $\mathcal{C}_{3}$. Every configuration $\{A, B, C, D\}$ from this class, see Figure 1, gives rise to twelve 4 -tuples counted by the first sum:

$$
\begin{aligned}
& (A, D, B, C),(A, D, C, B),(D, A, B, C),(D, A, C, B), \\
& (B, D, A, C),(B, D, C, A),(D, B, A, C),(D, B, C, A), \\
& (C, D, A, B),(C, D, B, A),(D, C, A, B),(D, C, B, A),
\end{aligned}
$$

and six 4-tuples counted by the second sum:

$$
(A, B, C, D),(A, C, B, D),(B, A, C, D),(B, C, A, D),(C, A, B, D),(C, B, A, D) .
$$

Hence, each configuration from $\mathcal{C}_{3}$, gives rise to twice as many 4-tuples (which are not 4-gons) that are counted by the first sum than those that are counted by the second sum.

It is harder to make similar comparisons for configurations coming from $\mathcal{C}_{i}$ where $i=0,1,2$. In order to do them, we further partition each of these classes into subclasses where the three collinear points belong to a fixed line, and the point off this line is also fixed. Namely, for each of these $i$, and for any line $x$ and any point $D$ not on $x$, let $\mathcal{C}_{i}^{(x, D)}$ denote the subset of $\mathcal{C}_{i}$ formed by all $\{A, B, C, D\}$ such that $A, B, C$ are on the line $x$, and $D$ is off the line $x$. Note that $d=d(x) \geq 3$. Let $\alpha$ be the number of points on $x$ collinear with $D$. Then $0 \leq \alpha \leq d-1$. See Figure 2 .

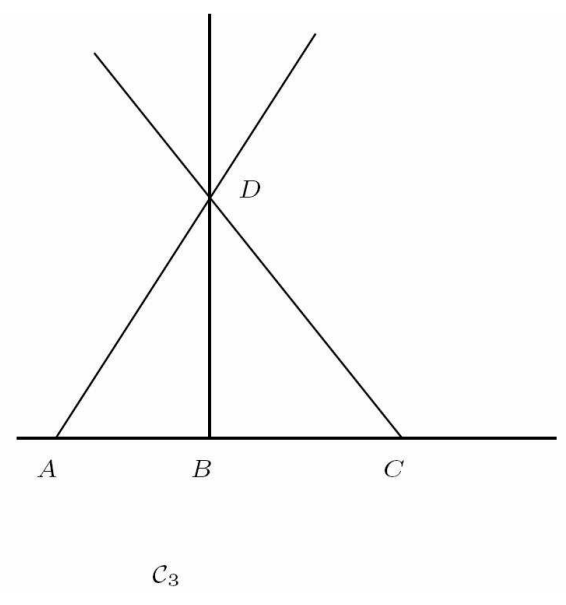

Figure 1

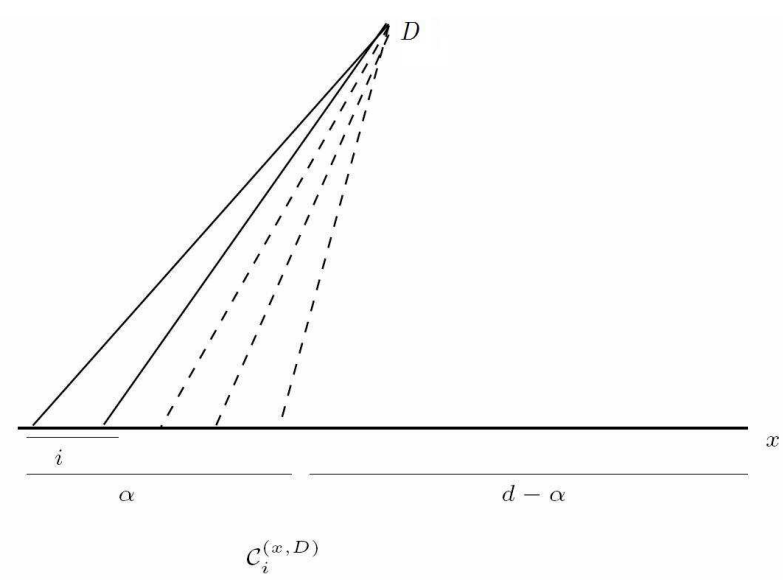

Figure 2

We obtain

$$
\left|\mathcal{C}_{0}^{(x, D)}\right|=\left(\begin{array}{c}
d-\alpha \\
3
\end{array}\right), \quad\left|\mathcal{C}_{1}^{(x, D)}\right|=\alpha\left(\begin{array}{c}
d-\alpha \\
2
\end{array}\right), \quad\left|\mathcal{C}_{2}^{(x, D)}\right|=\left(\begin{array}{l}
\alpha \\
2
\end{array}\right)(d-\alpha)
$$

It follows that all configurations from $\bigcup_{i=0}^{i=2} \mathcal{C}_{i}^{(x, D)}$, give rise to

$$
c^{(x, D)}:=6 \cdot\left(\left(\begin{array}{c}
d-\alpha \\
3
\end{array}\right)+\alpha\left(\begin{array}{c}
d-\alpha \\
2
\end{array}\right)+\left(\begin{array}{l}
\alpha \\
2
\end{array}\right)(d-\alpha)\right)
$$


4-tuples counted by the second sum.

For the same pair $(x, D)$, let us now count some special 4-tuples which are accounted in the first sum, and are not 4 -gons. Some of them arise from configurations $\mathcal{C}_{i}^{(x, D)}$, where $i=1$ or 2 , but others do not.

Let $\{D, X, Y, Z\} \in \mathcal{C}_{1}^{(x, D)}$ with $D \sim X,\{X, Y, Z\} \subseteq x$. Among all possible $(4 !=24)$ 4-tuples it gives rise to, $(D X Y Z)$ and $(D X Z Y)$ are the only ones with the property that

- $D$ is the first point,

- $D$ is collinear with the second point.

Clearly, these 4-tuples are counted in the first sum, they are not 4-gons, and there are $2 \cdot\left|\mathcal{C}_{1}^{(x, D)}\right|$ of them.

Similarly, let $\{D, X, Y, Z\} \in \mathcal{C}_{2}^{(x, D)}$ with $D \sim X, D \sim Y,\{X, Y, Z\} \subseteq x$. Among all 4 -tuples it gives rise to, the following six

$$
(D X Y Z),(D X Z Y),(D Y X Z),(D Y Z X),(X D Y Z),(Y D X Z)
$$

are the only ones with the property that

- $D$ is the first or the second point,

- the first point is collinear with the second point,

- the second point is collinear with the third point.

Clearly, these 4-tuples are counted in the first sum, they are not 4-gons, and there are $6 \cdot\left|\mathcal{C}_{2}^{(x, D)}\right|$ of them.

Hence, so far, we have found

$$
s(x, D):=2 \cdot\left|\mathcal{C}_{1}^{(x, D)}\right|+6 \cdot\left|\mathcal{C}_{2}^{(x, D)}\right|=3 \alpha(\alpha-1)(d-\alpha)+\alpha(d-\alpha)(d-\alpha-1)
$$

4-tuples counted by the first sum which are not 4-gons.

Finally, for the same fixed $x$ and $D$, we consider all 4-tuples $(X, Y, D, Z)$ with the property that $X, Y \in x, Z \notin x$, and $D \nsim Y$. Note that, as $Z \notin x$, none of these 4-tuples arises from a configuration of $\bigcup_{i=0}^{3} \mathcal{C}_{i}^{(x, D)}$, all of them are counted in the first sum, none of them is a 4-gon (as $D \not Y$ ), and there are

$$
t(x, D):=(d-\alpha)(d-1)(n-d-1)
$$

of them: first choose point $Y$ on $x$, then $X \in x$, then $Z \notin x$ and different from $D$.

At this point it is important to remark that, because of the specific 4-tuples we counted, no 4-tuple accounted in $s(x, D)$ or $t(x, D)$ is also accounted in $s(y, E)$ or $t(y, E)$, unless $(x, D)=(y, E)$. Indeed, suppose $(K, L, M, N)$ were such a 4 -tuple. If $L \sim M$, then this 4-tuple is counted by $s(x, D)$ and $s(y, E)$. In this case, $x=M N=y$. As $D$ and $E$ have 
to be the only point off this line, $D=E$. If $L \neq M$, then $(K, L, M, N)$ is counted by $t(x, D)$ and $t(y, E)$. In this case, $x=K L=y$, and $D=M=E$.

Hence, in order to prove our lemma it suffices to show that

$$
s(x, D)+t(x, D)-2 c^{(x, D)} \geq 0 .
$$

As $d \geq 3$, this is equivalent to

$$
n \geq 3 d+\alpha-3-\frac{\alpha}{d-1}
$$

As $\alpha \leq d-1$, we obtain that (3) is satisfied for $n \geq 4 d-5$. By Lemma 2.2 this is the case, and the proof of our lemma is finished.

Remark The end of the proof above requires $\Delta \leq n / 4+1$. One may wonder why then in the statement of Lemma 2.2 we used $\Delta<n / 4$. The reason we choose to proceed as we did is that changing the upper bound on $\Delta$ in Lemma 2.2 to $n / 4+1$ does not decrease the lower bound on $n$, namely, 157.

The sums in the right hand side of (2) can be combined as

$$
\begin{gathered}
\frac{1}{8} \cdot\left[\sum_{x \in \mathcal{L}} d(x)_{(2)}(n-d(x))_{(2)}-2 \sum_{x \in \mathcal{L}} d(x)_{(3)}(n-d(x))\right]= \\
\frac{1}{8} \sum_{x \in \mathcal{L}} d(x)_{(2)}(n-d(x))(n-3 d(x)+3) .
\end{gathered}
$$

We begin with maximizing this sum over all partial planes with $n$ points and $n$ lines, or, equivalently, over their 4-cycle-free $n$ by $n$ bipartite Levi graphs. Most proofs of (1) follow from the fact that no pair of points is on two lines, which implies

$$
\sum_{x \in \mathcal{L}}\left(\begin{array}{c}
d(x) \\
2
\end{array}\right) \leq\left(\begin{array}{l}
n \\
2
\end{array}\right)
$$

This suggests to consider the related optimization problem over the reals.

For $n \geq 1$, let

$$
D=\left\{\mathbf{x}: \mathbf{x}=\left(x_{1}, \ldots x_{n}\right) \in \mathbb{R}^{n}, x_{i} \geq 1 \text { for all } i, \text { and } \sum_{i=1}^{n}\left(\begin{array}{c}
x_{i} \\
2
\end{array}\right) \leq\left(\begin{array}{c}
n \\
2
\end{array}\right)\right\}
$$

Let $f(t)=t(t-1)(n-t)(n-3 t+3)$. For $\mathbf{x} \in D$, let $F(\mathbf{x})=\sum_{i=1}^{n} f\left(x_{i}\right)$. As $D$ is compact, $\max _{\mathbf{x} \in D} F(\mathbf{x})$ exists. We are interested in determining it, together with all points in $D$ where it is attained.

Let $r_{n}=\frac{1}{2}(1+\sqrt{4 n-3})$ be the positive root of equation $n\left(\begin{array}{l}x \\ 2\end{array}\right)=\left(\begin{array}{l}n \\ 2\end{array}\right)$, and let $\mathbf{r}$ denote the point with all coordinates equal to $r_{n}$. Note that $\mathbf{r} \in D$. 
Lemma 2.4 Let $n \geq 21$. Then

$$
\max _{\mathbf{x} \in D} F(\mathbf{x})=F(\mathbf{r})=n f\left(r_{n}\right),
$$

and $\mathbf{r}$ is the only point of $D$ where the maximum is attained.

Proof. It is easy to check that $f$ changes its concavity on $[1, n / 4]$. Therefore, an approach which first comes to mind, namely using the Jensen's inequality, fails. To prove Lemma 2.4, we use the Kuhn-Tucker theorem [14].

Suppose functions $G, g_{1}, \ldots, g_{m}: \mathbb{R}^{n} \rightarrow \mathbb{R}$, are differentiable, and $E=\left\{\mathrm{x} \in \mathbb{R}^{n}\right.$ : $g_{i}(\mathbf{x}) \geq 0$ for all $\left.i=1, \ldots, m\right\}$. For $\mathbf{x} \in E$, let $I(\mathbf{x})=\left\{i: g_{i}(\mathbf{x})=0,1 \leq i \leq m\right\}$. If the gradients $\nabla g_{i}(\mathbf{x}), i \in I(\mathbf{x})$, are linearly independent, we say that the constraints $g_{i}$, $i=1, \ldots, m$, satisfy the $L I$ regularity condition at $\mathbf{x}$. Then the following theorem holds (See Section 5.3.1 from Aoki [1]).

Theorem 3 Suppose functions $G, g_{1}, \ldots, g_{m}: \mathbb{R}^{n} \rightarrow \mathbb{R}$, are continuously differentiable, and $E=\left\{\mathbf{x} \in \mathbb{R}^{n}: g_{i}(\mathbf{x}) \geq 0\right.$ for all $\left.i=1, \ldots, m\right\}$. If

$$
\max _{\mathbf{x} \in E} G(\mathbf{x})=G(\mathbf{z}) \text { for some } \mathbf{z} \in E,
$$

and the LI regularity condition at $\mathbf{z}$ is met, then there exist real numbers $\lambda_{i} \geq 0$, such that, for all $i=1, \ldots, m$,

(i) $\lambda_{i} g_{i}(\mathbf{z})=0$, and

(ii) $\nabla G(\mathbf{z})+\sum_{i=1}^{m} \lambda_{i} \nabla g_{i}(\mathbf{z})=0$.

In order to apply the theorem to our problem, we take $E=D, G=F, m=n+1$, $g_{1}(\mathbf{x})=\left(\begin{array}{c}n \\ 2\end{array}\right)-\sum_{i=1}^{n}\left(\begin{array}{c}x_{i} \\ 2\end{array}\right)$, and $g_{i}(\mathbf{x})=x_{i-1}-1$ for $i=2, \ldots, n+1$. It is easy to see that for every point of $E$, the LI regularity condition is satisfied. It follows from the facts that for $2 \leq i \leq n+1, \nabla g_{i}(\mathbf{x})$ is a vector from the standard basis of $\mathbb{R}^{n}$ having 1 as its $(i-1)$ th component, and $\nabla g_{1}(\mathbf{x})=\left(1 / 2-x_{1}, 1 / 2-x_{2}, \ldots, 1 / 2-x_{n}\right)$. If $i \in I(\mathbf{x})$, and $i>1$, then the $(i-1)$ th component of $\nabla g_{1}(\mathbf{x})$ is $-1 / 2$. If $1 \in I(\mathbf{x})$, then there must be a nonzero $j$ th component of $\nabla g_{1}(\mathbf{x})$ for some $j \notin I(\mathbf{x})$, as $\sum_{j \notin I(\mathbf{x})}\left(\begin{array}{c}x_{j} \\ 2\end{array}\right)=\left(\begin{array}{c}n \\ 2\end{array}\right)$.

Therefore, by Theorem 3 , for a point $\mathbf{z}=\left(z_{1}, \ldots, z_{n}\right)$ of the absolute maximum of $F$ over $D$ there exists $\lambda, \lambda_{1}, \ldots, \lambda_{n} \geq 0$ such that

$$
\begin{gathered}
\lambda\left(\left(\begin{array}{l}
n \\
2
\end{array}\right)-\sum_{i=1}^{n}\left(\begin{array}{c}
z_{i} \\
2
\end{array}\right)\right)=0, \\
\lambda_{i}\left(z_{i}-1\right)=0, \quad i=1, \ldots, n, \\
f^{\prime}\left(z_{i}\right)-\lambda\left(z_{i}-\frac{1}{2}\right)+\lambda_{i}=0, \quad i=1, \ldots, n .
\end{gathered}
$$

Our goal is to prove that $\mathbf{z}=\mathbf{r}$. 
Let $N=n / 3+1$. As roots of $f$ on $[1, n]$ are $1, N$, and $n, f(t)>0$ on $(1, N)$, and $f(t) \leq 0$ on $(N, n)$. It is easy to check that $\max _{[1, n]} f(x)$ is attained at only one point $M \in(n / 5, n / 4)$, and $f$ is increasing on $[1, M]$ and decreasing on $[M, N]$.

We begin by showing that $z_{i}<n / 4$ for all $i=1, \ldots, n$. Suppose this is not the case, and $z_{j} \geq n / 4$ for some $j$. Then, from $(4), z_{j} \leq n$.

If $z_{j} \in[N, n]$, then $f\left(z_{j}\right) \leq 0$. In this case $f(2)>f\left(z_{j}\right)$. Therefore, changing $z_{j}$ in $\mathbf{z}$ to 2, gives another point $\mathbf{z}^{\prime} \in D$ with $F\left(\mathbf{z}^{\prime}\right)>F(\mathbf{z})$, a contradiction.

If $z_{j} \in(M, N)$, then there exists a point $z_{j}^{\prime} \in(1, M)$ such that $f\left(z_{j}^{\prime}\right)=f\left(z_{j}\right)$. Therefore, changing $z_{j}$ in $\mathbf{z}$ to $z_{j}^{\prime}$, gives another point $\mathbf{z}^{\prime} \in D$ with $f\left(\mathbf{z}^{\prime}\right)=f(\mathbf{z})$. It is also clear that $\left(\begin{array}{c}z_{j}^{\prime} \\ 2\end{array}\right)<\left(\begin{array}{c}z_{j} \\ 2\end{array}\right)$. Therefore there exists an $\epsilon>0$ such that, for $z_{j}^{\prime \prime}=z_{j}^{\prime}+\epsilon, f\left(z_{j}^{\prime \prime}\right)>f\left(z_{j}^{\prime}\right)$. Replacing $z_{j}^{\prime}$ in $\mathbf{z}^{\prime}$ by $z_{j}^{\prime \prime}$, we obtain a point $\mathbf{z}^{\prime \prime} \in D$ such that $F\left(\mathbf{z}^{\prime \prime}\right)>F(\mathbf{z})$, a contradiction again. Therefore $z_{i} \leq M<n / 4$ for all $i=1, \ldots, n$.

Next we show that $\lambda_{i}=0$ for all $i=1 \ldots, n$. Suppose, for some $k \geq 1$, exactly $k$ of $\lambda_{i}$ are positive, and others are zeros. Without loss of generality, we may assume $\lambda_{1}>0, \ldots, \lambda_{k}>0$, and $\lambda_{k+1}=\ldots=\lambda_{n}=0$. Then, from (6), $z_{1}=z_{2}=\ldots=z_{k}=1$. Clearly, $k \leq n-1$, as otherwise $F(\mathbf{z})=0$, and $\mathbf{z}$ is not a point of the absolute maximum. Then, from (7), we get

$$
\begin{gathered}
f^{\prime}(1)-\lambda \cdot \frac{1}{2}+\lambda_{i}=0 \text { for all } i=1, \ldots, k, \text { and } \\
\lambda=\frac{f^{\prime}\left(z_{i}\right)}{z_{i}-1 / 2} \text { for all } i=k+1, \ldots, n .
\end{gathered}
$$

As $f^{\prime}(1)=n(n-1)>0$, and $\lambda_{1}>0,(8)$ implies $\lambda>0$. The derivative of the function $h: t \rightarrow f^{\prime}(t) /(t-1 / 2)$ is

$$
h^{\prime}(t)=12(2 t-n-1)-\frac{n}{(2 t-1)^{2}},
$$

and it is negative on $[1, n / 4)$. Therefore $h$ is decreasing on $[1, n / 4)$, and (9) implies that all $z_{i}, i=k+1, \ldots, n$, are equal.

Let $a=a(n, k)$ be their common value. As $a \geq 1$, from (5) we obtain

$$
(n-k)\left(\begin{array}{l}
a \\
2
\end{array}\right)=\left(\begin{array}{l}
n \\
2
\end{array}\right), \quad \text { or } \quad a=\frac{1}{2}+\sqrt{\frac{1}{4}+\frac{n(n-1)}{n-k}} .
$$

Hence

$$
F(\mathbf{z})=(n-k) f(a) .
$$

Consider a function $u_{n}(k)=(n-k) f(a), k \in[1, n / 4)$. It is a straightforward verification that

$$
\frac{\partial u}{\partial k}=-\frac{n^{2}(n-1)^{2}}{(n-k)^{2} R}(4 n-3 R), \text { where } R=\sqrt{1+\frac{4 n(n-1)}{n-k}} .
$$

As $R<4 n / 3$ on $[0, n / 4), \partial u / \partial k<0$ on $[0, n / 4)$. Hence

$$
u_{n}(1)=\max \left\{u_{n}(k), k \in\{1,2, \ldots, n-1\}\right\} .
$$


But it is easy to see that $u_{n}(0)>u_{n}(1)$ for all $n \geq 1$. This contradicts the assumption $k \geq 1$. Hence $\lambda_{i}=0$ for all $i=1, \ldots, n$.

Then (9) holds for all $i=1, \ldots, n$, and so all $z_{i}$ are equal. By (5), their common value is $r_{n}$. Hence $F$ attains its maximum on $D$ at $\mathbf{x}=\mathbf{r}$ only.

We return to the proof of Theorem 1 . If $\delta(G) \geq 1$, then $G$ is the Levi graph of a partial plane. As $r_{n_{q}}=q+1$, by (2) and Lemma 2.4, we obtain

$$
c_{8}(G)=\leq \frac{1}{8} \sum_{x \in \mathcal{L}} f(d(x)) \leq n_{q} f\left(r_{n_{q}}\right)=\left(\begin{array}{c}
n_{q} \\
2
\end{array}\right)\left(\begin{array}{c}
q \\
2
\end{array}\right)^{2}=c_{8}\left(G\left(\Pi_{q}\right)\right) .
$$

If $c_{8}(G)=c_{8}\left(G\left(\Pi_{q}\right)\right)$, then every line contains $q+1$ points. Hence the graph is of the maximum size $(q+1) n_{q}$ among all $n_{q}$ by $n_{q}$ bipartite 4 -cycle-free graphs. As we explained in the introduction, it is known that in this case the graph must be isomorphic to a $G\left(\Pi_{q}\right)$.

If $G$ contains isolated vertices, let $G^{\prime}$ be a graph obtained from $G$ by connecting each isolated vertex of $G$ by one edge to a vertex in the other partition of $G$. Then $G^{\prime}$ is an $n_{q}$ by $n_{q}$ bipartite graph of girth at least six, $\delta\left(G^{\prime}\right)=1, c_{8}(G)=c_{8}\left(G^{\prime}\right)$, and $G^{\prime}$ is the Levi graph of a partial plane with $n_{q}$ points and $n_{q}$ lines. By the argument above, $G^{\prime}$ is isomorphic to a $G\left(\Pi_{q}\right)$, and $\delta\left(G^{\prime}\right)=q+1>1$, a contradiction. This completes the proof of Theorem 1.

\section{Concluding remarks}

The general problem of determining the maximum value of $c_{2 k}(G)$ for $n$ by $n$ bipartite graphs $G$ of girth at least $g$ appears to be difficult, especially when $g$ is small relative to $k$. We believe that a result similar to Theorem 1 holds for two other generalized polygons or order $(q, q)$.

Conjecture 5 Let $k \in\{4,6\}$, and $n=n_{q}^{k}$. Let $G$ be an $n$ by $n$ bipartite graph of girth at least $2 k$, and suppose $\Pi_{q}^{k}$ exists. Then, for sufficiently large $q$,

$$
c_{2 k+2}(G) \leq c_{2 k+2}\left(G\left(\Pi_{q}^{k}\right)\right),
$$

with equality if only if $G=G\left(\Pi_{q}^{k}\right)$.

We also believe that a much stronger statement holds.

Conjecture 6 Let $k \in\{3,4,6\}$, and $n=n_{q}^{k}$. Let $G$ be an $n$ by $n$ bipartite graph of girth at least $2 k$, and suppose $\Pi_{q}^{k}$ exists. Then, for every $t \geq k$, and for sufficiently large $q$,

$$
c_{2 t}(G) \leq c_{2 t}\left(G\left(\Pi_{q}^{k}\right)\right),
$$

with equality if and only if $G=G\left(\Pi_{q}^{k}\right)$ for some generalized polygon $\Pi_{q}^{k}$.

As we have seen, this statement is correct for $(k, t) \in\{(3,3),(3,4),(4,4),(6,6)\}$. We have not been able to settle Conjecture 6 even for $k=3$. 


\section{Acknowledgment}

We wish to thank the anonymous referee for pointing to some small errors in the original presentation. A large part of this article was written when the first author was visiting the Department of Mathematical Sciences of the University of Delaware, and when the second author was visiting the Department of Mathematics of the University of California at San Diego and the University of Ghent. We are grateful to these departments for providing us with excellent working conditions and for their hospitality.

\section{References}

[1] M. Aoki, Introduction to Optimization Techniques: fundamentals and applications of nonlinear programming, Macmillan New York, 1971.

[2] B. Bollobás, Extremal Graph Theory, Academic Press, London, 1978.

[3] B. Bollobás, Modern Graph Theory, Springer-Verlag New York, Inc., 1998.

[4] A. E. Brouwer, A. M. Cohen, A. Neumaier, Distance-Regular Graphs, SpringerVerlag, Berlin, 1989.

[5] P. Erdős, On some problems in graph theory, combinatorial analysis and combinatorial number theory. Graph theory and combinatorics (Cambridge, 1983), 1-17, Academic Press, London, 1984.

[6] W. Feit, G. Higman, The nonexistence of certain generalized polygons, J. Algebra 1, 1964, 114-131.

[7] G. Fiorini, F. Lazebnik, On a bound for the maximum number of $C_{8}^{\prime} s$ in a 4-cycle free bipartite graph Congressus Numerantium, 99 (1994), 191-197.

[8] G. Fiorini, F. Lazebnik, An Extremal Characterization of the Incidence Graphs of Projective Planes, Applicandae Matematicae, 52 (1998), 257-260.

[9] D. Fisher, The Number of Triangles in a $K_{4}$-Free Graph, Discrete Mathematics 69 (1989), 203-205.

[10] Z. Füredi, Turán type problems. Surveys in Combinatorics, 1991 (Guildford, 1991), 253-300, London Math. Soc. Lecture Note Ser., 166, Cambridge Univ. Press, Cambridge, 1991.

[11] S. Hoory, The size of bipartite graphs with a given girth, J. Combin. Theory Ser. B, 86(2) (2002), 215-220.

[12] E. Györi, On the Number of $C_{5}^{\prime} s$ in a Triangle-Free Graph, Combinatorica 9(1) (1989) 101-102.

[13] E. Györi, J. Pach, \& M. Simonovits, On the Maximum Number of Certain Subgraphs in $K_{r}$-Free Graphs, Graphs and Combinatorics 7 (1991), 31-37. 
[14] H. W. Kuhn, A.W. Tucker, Nonlinear programming. Proceedings of 2nd Berkeley Symposium: (1951) 481-492, Berkeley: University of California Press.

[15] S. Neuwirth, The size of bipartite graph with girth eight, ArXiv:math/0102210, 2001.

[16] C. P. Teo and K. M. Koh, The Number of Shortest Cycles and the Chromatic Uniqueness of a Graph, Journal of Graph Theory, Vol. 16, No. 1, (1992), 7-15.

[17] I. Reiman, Über ein Problem von K. Zarankiewicz, Acta. Math. Acad. Sci. Hungar. 9, (1958), 269-279.

[18] M. Simonovits, Extremal Graph Theory, in: Selected Topics in Graph Theory 2, L. W. Beineke and R. J. Wilson (eds.), Academic Press, London, 1983, 161-200.

[19] H. Van Maldeghem, Generalized polygons. Monographs in Mathematics, 93. Birkhuser Verlag, Basel, 1998. 